für diesen Specht als Ostgrenze den Tocantins an. Wie man sieht, erstreckt sich sein Verbreitungsgebiet jedoch über ganz N.0. Brasilien.

Campophilus trachelopyrus (Malh.) 13 Stück, Pará und Umgegend. Col. do Prata, ob. Guamá, Cussary bei Santarém.

Ceophloeus lineatus (L.) 20 Stück, Pará und Umgegend, Marajó, Mexiana, Maranao. Hier und auf Marajó der gemeinste Specht.

Picumnus amazonicus Sn. 10 Stück, Marajó, Monte Alegre, Cussary bei Santarém. S. Ornith. Monatsberichte 1906 S. 60. Ich habe seitdem den Vogel auch noch im Uferwald bei Monte Alegre gefunden.

In dieser Liste befinden sich also zwei neue Spechte (Chloronerpes nov. spec. und Picumnus amazonicus Sn.) und 6 aus der Gegend noch nicht bekannte, nämlich Colaptes campestris (Vieill.), Chrysoptilus punctigula (Bodd.), Chrysoptilus mariae Harg., Melanerpes candidus (Otto), Cerchneipicus tinnunculus (Wagl.), Campophilus melanoleucus (Gm.) (für Pará neu). Besonders interessant erscheint mir das, soviel ich weifs zum ersten Mal nachgewiesene, Vorkommen zweier Spechte aus dem südbrasilianischen Kamposgebiet, des Colaptes campestris (Vieill.) auf der kamposartigen Serra von Monte Alegre und des Melanerpes candidus (Otto) auf den Kampos von Marajó. Die Grenze des brasilianischen Kamposgebietes (ornithologisch) muls also jedenfalls weiter nach $\mathrm{N}$. gelegt werden, als man bisher anzunehmen geneigt war. Andere nach dem südbrasilianischen Kamposgebiet hinweisende verwandtschaftliche Beziehungen (z. B. Picolaptes bivittatus) werde ich später noch zu berühren haben.

(Fortsetzung folgt.)

\title{
Ein Beitrag zur Ornis des Tianschan.
}

\section{Von George Smallbones.}

Im Jahre 1900 unternahm Dr. G. von Almásy eine Expedition in den Tianschan, deren reiche ornithologische Ausbeute vom k. k. Naturhistorischen Hofmuseum in Wien erworben wurde. Ich wurde von Herrn Kustos Dr. von Lorenz mit der Bestimmung und Beschreibung dieser Collection betraut und sage ihm für alle Hilfe und Unterstützung, welche er mir bei dieser Arbeit gewährte, besten Dank. Die in [. . . .] gehaltenen Notizen über Vorkommen, Lebensweise etc. stammen von Herrn Dr. von Almásy, der die Güte hatte, sie mir zur Publikation zu überlassen und dem ich hierfür bestens danke.

Almásy sammelte im Quellgebiete des Issyk-Kul und zwar im östlichen Teile des Terskei Ala Tan, dann im oberen Sary Dschas-Tale, endlich am Kokdser einem Quelllaufe des Ili und 
in dem Reviere zwischen dem zum Tekes ziehenden Kapkak und zwischen Naryn Kol.

1. Erithacus rubeculus L. ơ 9. X. 1900 in Przewalsk.

[Wurde nur in dem erlegten Exemplar im Walde nahe bei Przewalsk beobachtet. Dürfte für das Gebiet nur ein seltener Durchzügler sein.]

2. Erithacus hafizi Severtz. o 11. V. 1900 Ilijsk.

[Gemeiner Brutvogel am mittleren und unteren Ili.]

3. Erithacus suecicus L. 1 के im Frühjahrskleide aus Ilijsk, 2 đo und 2 \% im Herbstkleide aus Przewalsk.

[Brutvogel des ganzen Gebietes, der Balchasch-Niederung sowohl, wo es sumpfige Bruchwiesen besiedelt, als auch der tundrenartigen Hochflächen am Sary-Dschas bis zu etwa $4000 \mathrm{~m}$ Meereshöhe, wo es in dem dichten Teppich einer spannenhohen Zwergweidenart nistet. Während des Zuges häufig in der Umgebung des Issyk-Kul.]

4. Ruticilla rufiventris (Vieill.) 1 o juv., August, Torpu Syrt. 5 đ ad. August.

5. Ruticilla caeruleocephala Vig. 6 के ad., 3 \% ad., 1 б juv. August, September und Oktober. Kapkak, Przewalsk, KarakolPrzewalsk, Ajuk-Tascz und Naryn.

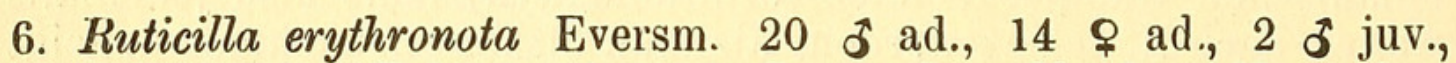
1 \& juv. Februar, August, September, Oktober, November von Karakol, Berkut-Szu, Kapkak, Przewalsk, KarakolPrzewalsk, Koj-Szari, Dzetiguz, Örtök, Ottuk-Tas, Csergalan-Basi, Naryn und Ajuk-Tascz.

7. Ruticilla erythrogastra Güld. 16 o ad., 1 \& ad., 1 ? August, September, Oktober, November, Dezember Ottuk-Tas, Eskili-Tas, Koj-Szary, Przewalsk, Torpu-Syrt, Terskei-AlaTau, Ajuk-Tascz.

8. Ruticilla erythrogastra grandis Gould. 1 o ad., 3 \% ad. August, September, Oktober Naryn, Terskei-Ala-Tau, Przewalsk.

[Während der Brutzeit besiedeln diese 3 erwähnten Ruticillen die hohen und höchsten Lagen des Tian-Schan bis an die Schneegrenze. Im Herbst und Winter in grofsen Mengen in den tieferen Lagen und den Ufern des Issyk-Kul.

9. Accentor collaris rufilatus Severtz. o ad. 1. XI. 1900 Przewalsk.

10. Accentor altaicus Brandt 2 o ad., 2 \% ad. Juli, August Karakol-Bas, Tsub-Basi.

11. Accentor fulvescens Severtz. $2 \%$ ad., 1 ? ad. Februar, September, November Kapkak, Przewalsk.

12. Accentor atrigularis Brandt 11 के ad., $6 \%$ ad. Juli, August, September, Oktober, November Karakol-Bas, Tsub-Basi, 
Ottuk-Tas, Naryn-Kol, Türgen, Dzetiguz, Örtök, Przewalsk, Naryn, Ajuk-Tascz.

[Die 4 erwähnten Accentores kommen in der Alpenmattenregion von $3000 \mathrm{~m}$ Meereshöhe aufwärts überall vor. Der Zahl nach am häufigsten ist Acc. atrigularis, welcher geradezu die typische Vogelerscheinung selbst der trostlosesten Hochgebirgsscenerie bildet. Zu Beginn des Winters streichen die Vögel in tiefere Lagen herab, scheinen aber die Issyk-Kul-Mulde selbst und die warmen Ufer des Sees nicht aufzusuchen.]

13. Saxicola isabellina Cretzschm. 2 б ad. August Torpu, Przewalsk.

[Nicht sehr häufig, im Tiën-Schan gesammelt auf der steppenartigen Hochfläche am Küljü-Su (ca. 3000 m) und - auf dem Zuge - in den Steppen am Issyk-Kul.]

14. Saxicola pleschanka (Lepech.) 1 o ad., 2 \% ad., o juv. Juli, August, September Terskei-Ala-Tau, Przewalsk.

[Nur am Südufer des Issyk-Kul (Steppengebiet) gesammelt.]

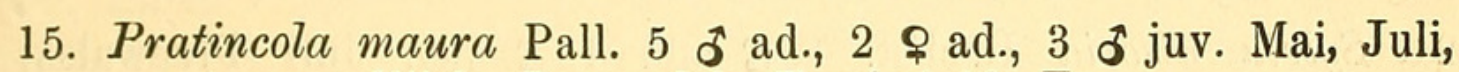
August Ilijsk, Przewalsk, Terskei-Ala-Tau.

[Gemein in der Ili-Niederung, ebenso auf den Wiesen und Feldern bei Przewalsk.]

16. Monticola saxatilis L. 1 ठ์ ad., 2 \% ad., 3 ठे juv. August Küljü-Szu, Terskei-Ala-Tau, Ajuk-Tascz, Naryn, Eskeli-Tas.

[Scheint überall an geeigneten Stellen vorzukommen, da wir ihn sowohl im Ili-Cannon, als auch bei etwa $3400 \mathrm{~m}$ Meereshöhe auf dem Syrts beobachteten.]

17. 'Turdus viscivorus bonapartei Cab. 2 oै ad., 2 \% ad., 2 juv.?, 1 ô juv., 1 \& juv. Februar, Juli, August, September Karakol-Bas, Dzergez, Naryn, Ajuk-Tascz, Przewalsk.

1 o und $2 \%$ aus Dzergez, Naryn und Ajuk-Tascz erlegt im September beziehungsweise August, sind auf der Unterseite bedeutend gelber als $T$. viscivorus typicus, rostgelb, die oberen Schwanzdecken gelb gesäumt; diesen 3 auffallend gelben Exemplaren gleicht ein $\hat{\delta}$ aus Innsbruck. Ein $\hat{\delta}$ aus Przewalsk im April erlegt ist bedeutend heller, viel weniger gelb, vielleicht weil es im Frühjahrskleid ist. Alle Exemplare, auch die jungen, unterscheiden sich von $T$. viscivorus typicus durch längere Schnäbel und Flügel.

[Häufiger Brutvogel im mittleren Waldgürtel des TerskeiAla-Tau. Während des Herbstzuges sehr gemein in den Dorngestrüppen am Issyk-Kul-Ufer.]

18. Turdus atrigularis Temm. 11 ऽ ad. Mai, August, Oktober, November Ilisk, Ajuk-Tascz, Koj-Szary, Dsetiguz, Przewalsk.

[Seltener Brutvogel in alten Pappelbeständen bei Ilijsk. Häufig im Waldgebiet des Tiën-Schan.] 19. Acrocephalus arundinaceus L. o ad. Juni Ilisk. 
[In den Röhrichten an der Talgoru, am Ili und am Tscharym überall, doch nirgends in grofser Anzahl.]

20. Acrocephalus dumetorum (Blyth.) 2 ऽ ad., 1 \& ad. Mai, Juni Ilisk.

[Ebendort, jedoch etwas häufiger als Acrocephalus arundinaceus $\mathrm{L}$.

21. Phylloscopus viridanus Swinh. 4 o ad., 1 9 ad. Mai, Juni, August Ilisk, Karakol-Bas, Terskei-Ala-Tau.

22. Phylloscopus humei Brooks 10 ठे ad., 8 \% ad., 1 ad. ? Mai, Juli, August, September Ilisk, Kacska-Szu, Karakol-Bas, Karakol-Tal, Kapkak, Naryn-Kol, Ajuk-Tascz, Naryn.

[Gemein in den Nadelwäldern; nicht eben selten auch als Brutvogel in den Weidengebüschen am Ili.]

23. Phylloscopus tristis Blyth. ơ ad. Oktober Koj-Szary. zügler? - ]

[Nur ein Exemplar am Issyk-Kul-Ufer gesammelt. Durch-

24. Sylvia nisoria Bechst. 1 ठै ad., 1 ठో juv. Juli Przewalsk, Kasczka-Szu.

25. Sylvia familiaris (Menetr.) 1 \& ad. Mai Kok-Dschigde.

[Nicht eben selten bei Ilisk und in den Tamarix- und Saxaul-Wüsten am Ili bei Tscharym.]

26. Sylvia fuscipilea Severtz. 4 ơ ad., 2 \% ad. Mai, Juli, August Ilisk, Przewalsk.

[Gemein in den Niederungen und häufig in der Umgebung von Przewalsk.]

27. Cinclus asiaticus Swains. 2 \% ad. Oktober, November Przewalsk, Dsetiguz.

[Nicht sehr häufig; in zunehmender Zahl überhaupt erst gegen den Herbst hin beobachtet, wo er in der Mulde des IssykKul-Beckens herabstieg. Scheint deshalb für das Gebiet entweder Wintergast zu sein, oder - allerdings auch nur in geringer Anzahl - nur die höchstgelegenen Kettenzüge zu bewohnen.]

28. Cinclus leucogaster Bp. 6 o ad., 3 ㅇ ad., 1 juv ?, 1 ? Juli, August, Oktober, November Karakol-Bas, Csergalan-Basi, Naryn, Issyk-Kul, Przewalsk.

[An allen geeigneten Bächen des Gebirges gewöhnlich.]

29. Troglodytes pallida Hume. 3 ơ ad. September, Oktober Kapkak, Karakol-Przewalsk,

[„Im Fichtenwald überall vorkommend."]

30. Regulus himalayensis Jard. 4 o ad. September, Oktober Kapkak, Örtök.

[Ebenfalls im Fichtenwald ziemlich häufig.]

31. Myophoneus temmincki Vig. \$ै ad. 22. VII. Karakol-Bascz. 
[Scheint ziemlich selten zu sein. Beobachtete ein einziges Paar im Felsen des Karakol-Tales bei Przewalsk, von wo auch das Belegexemplar stammt.]

32. Leptopoecile sophiae Severtz. 3 ठ̊ ad., 1 ô juv. Oktober, November Karakol, Przewalsk.

[Trotz eifrigster Bemühungen gelang es mir nicht, dieses Vögelchens während der Brutzeit habhaft zu werden, sämtliche Belegexemplare stammen aus dem Spätherbst und aus der unmittelbaren Umgebung von Przewalsk, wo sie sich in Gesellschaft anderer Herbstwanderer im Gesträuch herumtrieben. Im Fichtenwalde - wie es nach Ssewertzoffs Angaben wahrscheinlich erschien - brütet Leptopoecile bestimmt nicht; ich vermute, dals ihre Brutstellen in den Krummholzfeldern der "Articha" (Junniperus pseudosabina) oberhalb der Waldgrenze (3500-4000) gelegen sein dürften, von wo aus sie erst im Herbst in den tiefer gelegenen Waldgürtel hinabstreifen.]

33. Parus songarus Severtz. 4 ठ ad., 5 \& ad., 1 juv. ? Juli, August, September Kacska-Szu, Karakol-Bas, Kapkak, Ajuk-Tascz, Naryn.

[Sehr gemein im Nadelwald.]

34. Parus cyanus tianschanicus Menzb. 14 o ad.,. 3 \% ad. September, Oktober, November Naryn-Kol, Örtök, KarakolTal, Przewalks.

[Nicht eben häufiger Brutvogel im schütteren Weidengebüsch und Röhricht am unteren Sli; gemein in den Laubdjungels des Tekkes-Tales. Ungemein häufig zu Beginn des Winters in der Umgebung von Przewalsk und am Ufer des Issyk-Kul.]

35. Parus ater rufipectus Severtz. 13 oे ad., 8 ㅇa․, 1 \% juv., 1 juv. ? Juli, August, September, Oktober, November Karakol-Bas, Naryn-Kol, Csergalan-Basi, Kapkak, Ajuk-TasczNaryn, Karakol-Tal, Dsetiguz, Przewalsk.

[, Sehr gemein im Nadelwald."]

36. Sitta neumayeri tephronota Sharpe ơ ad.? 22. X. Przewalsk.

[Auffallend selten im ganzen Gebiet. Aufser dem Belegexemplare (22. X. 1900, Przewalsk) beobachtete ich einen einzigen Kleiber im Felsen des Berkut-Passes am 22. VIII. 1900 in etwa 3400 m Meereshöhe im Gebiete der Sary-Dschas-Syrt's.]

37. Zichodroma muraria L. 4 đ ad. August, Oktober, November Örtök, Przwalsk, Berkut-Szu.

[Eine häufige Vogelgestalt des Tiën-Schan, im Hochsommer bis über die Vegetationsgrenze hinaus noch bis etwa $4000 \mathrm{~m}$ Meereshöhe beobachtet.]

38. Certhia familiaris scandulaca Pall. 10 ad., 2 \% ad. Oktober November, Dezember Przewalsk, Kapkak.

[„Nicht eben häufig im Nadelwald."] 
39. Otocorys albigula Bp. 5 ơ ad., 6 \% ad., 1 б juv., 2 \% juv., 1 ? August, September, Oktober, November Eskili-Das, Naryn-Kol, Teskei-Ala-Tau, Naryn, Torpu, Przewalsk.

[Eine typische Erseheinung des ganzen Gebirges. Im Dezember bis in die Steppen bei Wjernyi herab.]

40. Melanocorypha calandra L. ô ad., \$ juv. Juni Ilisk.

[Ebenso häufig, wie die Alpenlerche im Hochgebirge, ist die Kalanderlerche in allen Steppen der Niederung bei Wjernyi. Im Issyk-Kul-Becken habe ich sie nicht beobachtet.]

41. Calandrella brachydactyla (Leisl.) 2 o ad., $1 \subsetneq$ ad., 1 juv. ? Juni, August Ilisk, Torpu.

[Ebenso häufig in den Steppen, wie die vorige, scheint aber höher in das Gebirge aufzusteigen. Im Issyk-Kul-Becken und im Tekkes-Tal häufig. Ein Exemplar erlegte ich dann am 16. VIII. 1900 auf dem Torpu-Syrt (Sary-Dschais-Gebiet) in etwa $3600 \mathrm{~m}$ Meereshöhe.]

42. Alauda arvensis L. 3 \% ad., 1 đ juv., 1 ad. ? September, Oktober Naryn-Kol, Przewalsk.

[Auffallenderweise traf ich die ersten Lerchen erst im Tekkes-Tal bei Naryn-Kol im September, ohne vorher ihnen irgendwo begegnet $\mathrm{zu}$ sein. Später im Herbst stellten sich auch in Przewalsk Lerchen in geringer Anzahl ein.]

43. Anthus campestris L. 2 o ad., 9 ad. Juli, August KacskaSzu, Türgen, Przewalsk.

[Ziemlich häufig in allen Lehmsteppen des Tieflandes, fehlt auch an geeigneten Stellen des Tiën-Schan nicht, so bis in die Tschi-Steppen (Lasiogrostis splendens) des Sary-Dschas-Tales in etwa $3000 \mathrm{~m}$ Meereshöhe hinauf.]

44. Anthus spinoletta blakistoni Swinh. 5 o ad., $\$$ ad., ad. ? Juli, August, September Karakol-Bas, Tjub-Basi, Torpu Syrt, Naryn-Kol, Kapkak, Ajuk-Tasez.

[An allen Quellenmooren des Gebirges gemein.]

45. Anthus arboreus Bechst. of ad., \& ad. Juli, August Naryn-Kol, Ajuk-Tascz.

[,Häufig im Nadelwald."]

46. Motacilla alba L. $\delta$ ad., $\$$ ad., 2 б juv. Mai, Juli, September Ilisk, Karakol, Adr-Tör, Naryn.

[In den Niederungẻn des Ili nicht selten, während dort $M o t$. personata $\mathrm{zu}$ fehlen scheint.]

47. Motacilla personata Gould. 7 đad. März, Juli, Oktober Przewalsk, Karakol, Kacska-Szu, Karkara.

[In Tiën-Schan allüberall häufig, vertritt dort vollständig Mot. alba.] 
48. Motacilla melanope Pall. 5 o ad., $\mathbf{Q}$ ad., ô juv., $\mathbf{Q}$ juv. April, Juli, August, September Karakol-Bas, Tjub-Basi, Dsergez, Berkut-Szu, Karakol, Ilisk.

[Gemein an allen Bächen des Gebirges bis $3000 \mathrm{~m}$ Seehöhe.]

49. Motacilla citreola Pall. 4 o ad., 3 @ ad. Mai, Juni, Juli, August, November Ilisk, Karakol, Przewalsk, Tjub.

[Gemein in den Niederungen am Ili, nicht eben selten im Issyk-Kul-Becken.]

50. Budytes melanocephalus Lichtst. 4 o ad. Mai, Juni Ilisk.

[Zahlreicher Brutvogel in den Ili-Niederungen, an Menge der Feldeggsbachstelze überlegen. Typische Flora beobachtete ich während der Reise überhaupt nicht].

51. Emberiza melanocephala Scop. $\mathbf{9}$ ad. Juli Karakoltal.

j2. Emberiza buchanani Blyth. ô ad. August Ajuk-Tasez.

53. Emberiza leucocephala Gm. 2 ô ad. September Koj-Szary, Dsetiguz.

54. Emberiza cioides Brandt. 3 ô ad., 2 ㅇa., 2 ô juv. August, Oktober, November Karakol-Tal, Przewalsk.

Zwei o ad. haben stark abgestofsenes Gefieder, wodurch das braune Brustband sehr deutlich ohne weilse Federkanten hervortritt.

55. Emberiza cia stracheyi Moore. ô ad., 우 ad. Juli Kacska-Szu.

đ mit schmalen schwarzen Schaftstreifen am Rücken und ziemlich lichter Unterseite und Rücken.

56. Emberiza luteola Sparrm. 8 đ̄ ad. Mai, Juni, Juli, August Ilisk, Przewalsk.

57. Emberiza citrinella L. ơ ad., 39 ad. 1 ad. ? November Przewalsk.

58. Emberiza cia godlewskii Tacz. 2 ō ad. Oktober, November Przewalsk, Karakol.

Im Vergleich zu den Exempiaren des k. k. Hofmuseums sind unsere beiden $\delta$ am Rücken und auf der Unterseite weniger rostbraun, Vorderbrust, Kehle und Hals lichter grau.

[In den Sliniederungen wurden auffallend wenig Ammern beobachtet; wirklich häufig war dort nur $E$. luteola, die aber auch bei Przewalsk nicht eben selten ist. $E$. cia oder die derselben nahestehenden Formen sind in den tieferen Lagen des Tiën-Schan sehr zahlreich vertreten. Nächst bäufig ist $E$. leucocephala, während E. citrinella nur Wintergast des ganzen Gebietes zu sein scheint.]

59. Loxia curvirostra L. đa ad. September, Türgen.

[Dürfte in den Wäldern des centralen Tiën-Schan kaum regelmäfsiger Brutvogel sein, sondern eher nur ein sporadischer Gast aus nördlichen Gebieten.] 
60. Mycerobas carneiceps Hodgs. 4 के ad., 1 ठే juv. September, Dezember Zergalan-Asu, Karakol-Wald.

[Häufig in den höher gelegenen Wäldern.]

61. Carpodacus severtzovi Sharpe ơ ad. November Przewalsk.

[Vermutlich nur Wintergast, da früher nicht beobachtet.]

62. Carpodacus rhodochlamys (Brandt) 4 o ad., 2 \% ad. Juli, Oktober, November Przewalsk, Karakol-Bas, Örtök.

63. Carpodacus erythrina Pall. 9 đे ap, 4 \% ad., 2 б juv. Mai Juli, August, September Ilisk, Karakol, Karakol-Tal, Karakol-Bas, Tjub-Basi, Karagaity.

[Gemein sowohl in der Niederung an geeigneten Stellen, als auch im Gebirge bis an die obere Waldgrenze überall.]

64. Uragus sibirica Pall. 4 ơ ad., 2 \& ad. November, Dezember, Jänner Karakol-Tal, Przewalsk.

[Vermutlich nur Wintergast.]

65. Serinus pusillus Pall. 16 के ad., $4 \%$ ad., $1 \delta$ juv., 5 ad. ? Februar, April, Juli, Oktober, November Przewalsk, Karakol, Karakoltal, Karakol-Bas, Issyk-Kul.

[Gemeiner Brutvogel der Waldregion, zieht im Winter bis auf $2000 \mathrm{~m}$ Meereshöhe herab.]

66. Acanthis carduelis major Tacz. 7 के ad., 2 \& ad. November Przewalsk.

67. Acanthis caniceps caniceps Vig. 4 oे ad., \$ juv. Juli, Oktober, November Karakol-Tal, Przewalsk.

[Beide Formen sehr häufige Brutvögel der unteren Waldregion; kamen zu Beginn des Winters zahlreich in die Gärten und in die Dorngestrüppe am Issyk-Kul.]

68. Acanthis cannabina tringillirostris Bp. u. Schleg. o ad. Juli Zergalan.

[Hänflinge brüten ziemlich zahlreich im westlichen Teil des Issyk-Kul-Beckens zwischen den Getreidefeldern.]

69. Fringilla montifringilla L. đo ad. Oktober Przewalsk.

[Vermutlich nur Wintergast des Gebietes.]

70. Montifringilla brandti Bp. 4 ad. ? November, Jänner Przewalsk.

71. Montifringilla altaica Eversm. ơ ad., 2 \& ad., 1 ? Juli, August Karakol-Tal, Karakol-Bas.

[Montifringilla altaica ist ein verbreiteter, wenn auch nicht eben häufiger Brutvogel der hohen Gebirgskämme von der oberen Holzgrenze an bis nahe zur Schneegrenze. Mont. brandti wurde $\mathrm{zu}$ vorgerückter Jahreszeit erlegt, wo die Hochgebirgsvögel in die Talebenen (Issyk-Kul-Becken) herabstreichen.

72. Passer domesticus indicus Jard. u. Selby. 3 o ad., 4 \% ad. Juli, August, Oktober Ilisk, Przewalsk. 
73. Passer montana dilutus Richmond. $\delta$ ad. Juli Przewalsk.

[Zufälligkeiten während der Reise verhinderten leider ein ausgibiges Sammeln der interessanten Sperlingsformen, welche die Saksaul-Steppen und Pappelwälder der Niederungen besiedeln. Die mitgebrachten Exemplare stammen ausschliefslich aus der unmittelbaren Nähe der Ansiedlungen von Ilisk und von Przewalsk und stellen mithin nur ein halbdomesticiertes, gewifs auch eng an die recenten menschlichen Niederlassungen gebundenes Material dar.

74. Sturnus vulgaris porphyronotus Sharpe. 2 od., $4 \%$ ad., 4 ad. ? April, Juli, Oktober Koj-Szary, Przewalsk, Karakol, Karakol-Tal.

75. Pastor roseus L. 3 o ad. Mai, Juni Ilisk.

[In Scharen, die nach Hunderttausenden zählen, in den Niederungen und Steppen am unteren und mittleren Ili. Gewaltige Brutkolonien befinden sich in den Felsen des Ili-Cañon unterhalb Ilisk's. Brüten jedoch auch zahlreich in allen Wäldchen, wo alte Toghrok (Populus diversifolia) stehen. Der Rosenstar erfreut sich sowohl bei den russischen Ansiedlern als auch bei den einheimischen Nomaden als Heuschreckenvertilger eines weitgehenden Schutzes, so dals die Erlegung des Vogels nicht gerne gesehen wird.]

76. Pyrrhocorax pyrrhocorax L. 2 od ad, $\$$ ad. August OttukTas, Issyk-Kul, Akszu-Quellen.

77. Pyrrhocorax graculus L. $\%$ ad., o juv. August, September Karakol-Bas, Karagaity.

[Alpenkrähe und Alpendohle sind ständige Erscheinungen der höchsten Regionen des Hochgebirges.]

78. Nucitraga caryocatactes rothschildi Hartert. 2 o ad., 9 \% ad., 2 juv. ? März, Juli, August, September Terskei-Ala-Tau, Kacska-Szu, Karakol-Bas, Naryn-Kol. Ajuk-Tascz, Naryn, Przewalsk.

Die Schnabelformen variieren sehr und zeigen bald die Form von N. c. caryocatactes und bald die von N. c. macrorhynchus. In der dunkeln Färbung des Kleingefieders gleichen die angeführten Tian-Schan-Exemplare je einem Exemplar aus Sarajevo, aus Wagstadt in Schlesien und aus der Collection von Kronprinz Rudolf, leider ohne Fundort, unterscheiden sich jedoch von diesen 3 Stücken durch die von Hartert in ,Die Vögel der palaearktischen Fauna" p. 27, 45 erwähnten tief blauschwarzen Schwanz- und Steuerfedern sehr deutlich. [N. c. rothschildii kommt in allen Nadelwäldern ungemein zahlreich vor.]

79. Pica pica L. 2 ơ ad., 1 juv. ? Juli Santuscz-Pals, Przewalsk. Eine unserer europäischen Elster gleiche Form, doch mit weniger Metallglanz auf den Steuerfedern.

80. Colaeus monedula collaris Drummond ơ ad., 9 ad., juv. ? Mai, Juni, Ilisk. 
od. und \% ad. gleichen vielen Exemplaren aus NiederÖsterreich; juv. ? hat nur einen Anflug des weifsen Halsstreifens. Im ganzen sind unsere 3 Tianschanexemplare am Rücken, Bauch und Hals dunkler als solche aus Nied.-Österr., unter denen sich zahlreiche Stücke ohne weifsen Halsfleck finden, also Colaeus monedula spermologus (Vieill.).

81. Corvus cornix sharpii Oates ô ad. Oktober. Örtök.

Vollkommen wie Corvus cornix cornix L., nur sind alle grauen Partien des Gefieders bedeutend heller.

82. Corvus corax L. ơ ad., \$ juv. August Tjub-Asu, Terskei-Ala-Tau. 83. Corvus corone L. ठ juv. Juli. Przewalsk.

[,Sowohl Elstern, als auch die Dohlen, Raben und Krähen sind im ganzen Gebiet verbreitet."]

84. Lanius phoenicuroides romanowi Severtz. 2 of ad., 9 juv., 1 juv. ? Juni, Juli. Ilisk, Przewalsk.

Die beiden $\hat{\delta}$ ad. zeigen die für $\boldsymbol{L} . p h$. romanowi charakteristische Unterseite, Oberseite und zwar Kopf und Rücken sind wie bei $L$. isabellinus gleich braunlich grau. Das eine of ad. hat auch auf der Unterseite einen rosig gelblichen Schimmer, jedoch sind bei beiden die Primärschwingen ganz charakteristisch dunkel für $L . p h$. romanowi.

85. Lanius isabellinus Ehrenb. 4 ơ ad, 2 ơ juv. Mai, Juli, August, September. Ilisk, Karakol, Przewalsk, Karagaity.

Diese sechs Exemplare bilden ebenfalls einen Übergang zwischen $L$. isabellinus und $L$. ph. romanowi, da sie zwar die rosig gelbliche Unterseite von $L$. isabellinus, die dunklen Primärschwingen jedoch und 2 a ad. den rotbraunen Kopf von $L$. ph. romanowi haben. Es dürften $\mathrm{zwischen} \boldsymbol{L}$. isabellinus und $\boldsymbol{L}$. phoenicuroides zahlreiche Zwischenformen vorhanden sein, umsomehr als beide in denselben Gebieten vorkommen.

[In der Niederung sind beide Formen überall und im Gebirge an buschreichen Stellen bis an die obere Holzgrenze verbreitet. An Ort und Stelle konnte eine Unterscheidung der beiden Formen überhaupt nicht bewerkstelligt werden.]

86. Lanius minor Gm. \& ad. Mai Ilisk.

[In der Niederung selten, im Gebirge überhaupt nicht.]

87. Lanius mollis Eversm. ơ juv. September Türgen.

[,Im ganzen Gebiete selten."]

88. Muscicapa grisola 2 ơ ad. Mai, August. Ilisk, Terskei-Ala-Tau.

[An geeigneten Stellen überall zahlreich.]

89. Hirundo rustica L. 2 क ad., 2 \& ad. Juni Juli. Przewalsk.

Je ein $\delta$ und $\$$ mit rötlicher und je ein $\delta$ und $\$$ mit weifser Unterseite.

90. Chelidonaria urbica L. ठै ad., 3 \& ad. August. Karakol-Tal, Naryn. 
[Die Mehlschwalbe hat sich im Siebenstromlande noch nicht zu jener Symbiose mit dem Menschen herbeigelassen, welche wir in Europa zu sehen gewohnt sind. Die Brutkolonien derselben fanden sich - weit von jeder menschlichen Ansiedlung entfernt - entweder in Lösswänden, ähnlich den Kolonien der Uferschwalbe angelegt, oder noch häufiger in spaltenreichen Felswänden, wie bei den Mauerseglern, oft sogar mit diesen zusammen an einer Örtlichkeit. - - ]

91. Micropus pekinensis Swinh. $\mathbf{O}$ ad. September. Karagaity.

[Individuenreiche Kolonien finden sich häufig im Hochgebirge, sowohl in den Waldregionen, als auch in den Hochsteppen oberhalb derselben bis über $3000 \mathrm{~m}$ Meereshöhe.]

92. Dendrocopus leucopterus Salvad. ơ ad. Mai. Utogoi, Insel.

[Spärlich in den Pappel- und Weidenwäldern am unteren Sli.]

93. Picoides tridactylus L. 6 o ad., 3 ㅇa‥ 3 juv. ? Juli, August, September, Oktober. Ilisk, Naryn-Kol, Kapkak, AjukTasez, Naryn, Örtök, Bel-Kara-Szu.

[Gemein in den Nadelwäldern.]

94. Upupa epops L. o 5. VIII. Przewalsk.

Anscheinend ein junges Tier mit auffallend kurzem Schabel von nur 3,2 cm Länge, wie ihn nur noch ein ô meiner Sammlung vom 8. VII. 1904 aus Velm N.Ö. hat.

[,Im ganzen Gebiete selten."]

95. Surnia ulula tianschanica Smallbones 3 ô ad., $\mathbf{9}$ juv., juv. ? Kacska-Szu, Örtök, Dsergez, Przewalsk.

Diese 6 Exemplare wurden von mir in den Ornith. Monatsberichten 1906 No. 2 p. 27 als nova subspecies beschrieben.

[Sehr häufig im Nadelwald. Verhältnismälsig leicht zu beobachten, da sie zu jeder Tagesstunde sichtbar und überhaupt sehr beweglich ist.]

96. Scops scops ô ad. VI. Boguty-Tau.

[Im Wüstengebirge Boguty-Tau und am mittleren Tscharym in einigen Pärchen; an beiden Orten finden sich kleine Baumoasen inmitten vollständiger Wüste. Sonst nirgends beobachtet.]

97. Asio otus L. \& ad. 1 ? Juli, Jänner. Kacska-Szu, Przewalsk. Gleicht vollkommen einzelnen europäischen Exemplaren, deren Farbe jedoch untereinander stark variiert.

[Brutvogel der unteren Waldregion.]

98. Asio accipitrinus L. ơ ad., $\%$ ad. Juli, September. Karkara, Przewalsk.

Das Gefieder des ô ist bedeutend dunkler, mehr rostfarben, alle Farben schärfer ausgeprägt, das $\boldsymbol{Q}$ jedoch in der Färbung heller, speziell an den Flügeln uid am Schwanz mehr weifs. Diese auffallenden Erscheinungen sind jedenfalls Geschlechtsunterschiede. 
[Nicht eben seltener Brutvogel am Mittellauf der Gebirgsflüsse. Ihre Nistplätze liegen auf halbsumpfigen, weiten Schotterfeldern, sogenannten "Sasen".]

99. Bubo ignavus turcomanus Eversm. 2 đ’ ad., 2 \% ad. September, Oktober, Dezember; Naryn-Kol, Przewalsk.

Ein o und ein $\$$ vom September resp. Oktober sind im Grundton der Färbung dunkler braun als die bei den anderen Exemplaren, ebenfalls ot und $\$$ vom Oktober und Dezember, die inneren Enden der Schwanzfedern sind dunkler braun, die Querbinden der Unterflügeldecken sind breiter und mehr braun, die der äufseren Steuerfedern ebenfalls breiter und deutlicher, während bei den 2 letztgenannten Exemplaren die Querbinden der Unterflügeldecken und äufseren Steuerfedern schmal und hell sind. Das $\%$ vom Dezember aus Przewalsk ist besondeas auf der Unterseite am hellsten, ihm zunächst steht in Bezug auf den. Ton der Unterseite das vom Oktober aus Naryn-Kol, das den Übergang zu den erwähnten dunkleren Exemplaren bildet. Meiner Meinung nach haben die beiden dunkeln Exemplare noch das Sommerkleid, die beiden lichten bereits das Winterkleid.

[Bubo ignavus turcomanus Evers. ist häufig im Tiën-Schan, besonders in den Wäldern des Gebirges, scheint aber auch in der Ili-Niederung durchaus nicht selten zu sein.]

100. Falco sacer Gmel. ㅇ ad., 1 ad. ? Oktober. Przewalsk, KojSzary.

ad. ? dürfte nach der Grölse zu urteilen ein junges ô sein. Das $\%$ ad. zeigt in Bezug auf Färbung der Unterseite und des Kopf- und Rückengefieders Ähnlichkeit mit Falco hendersoni Hume, der nach der Ansicht Sharpe's im Brit. Cat. Birds I. p. 419 ein alter Falco sacer ist.

[Ziemlich häufiger Brutvogel, noch häufiger auf der Herbstwanderung. Steht als Beizvogel bei den Eingeborenen in hohem Ansehen.]

101. Falco hendersoni Hume. $\delta$ ad. August. Terskei-Ala-Tau. [Am oberen Naryn am 13. VIII. ein Exemplar gesammelt.]

102. Falco peregrinus Tunst. $\delta$ ad., $2 \%$ ad. Oktober. Przewalsk. Das $\delta$ ad. hat helltaubengraue Flügeldecken, untere Rückenfedern und Schwanzdecken, die Unterseite ist rosiggelb angehaucht; es stimmt in dieser Hinsicht in der Färbung und sonst im Allgemeinen mit den von Menzbier im Ibis 1884, p. 287 erwähnten Exemplar vom Menzaleh in Egypten, das er Falco leucogenys nennt, gut überein, doch weist keines unserer 3 Exemplare das von ihm für $\boldsymbol{F}$. leucogenys aufgestellte Kennzeichen, nämlich die gelbbraunen Federn unter dem Auge in deutlichem Gegensatz zum schmalen Bartstreifen auf.

„Falco peregrinus ist im ganzen Gebiet Brutvogel." 
103. Falco peregrinus babylonicus Gurney. \& juv. Nuvember. Naryn-Kol.

104. Falco cenchris Naum. ơ ad. Mai. Ilisk.

[In der Ebene am Ili nicht selten. In Przewalsk einigemale in der Gefangenschaft gesehen, dürfte im ganzen Gebiet sporadischer Brutvogel sein.]

105. Falco subbuteo L. 3 के ad., $\$$ ad., ฮิ juv. Mai, August, September, Oktober. Ilisk, Przewalsk, Akszu-Quellen.

[Überall recht häufig.]

106. Falco aesalon Tunst. đo ad., đo juv. September, November.

Przewalsk, Issyk-Kul.

[Nicht seltener Brutvogel im Hochgebirge im Einzugsgebiet des Sary-Dschas. Sehr häufig während der Herbstwanderung. Wird als Beizvogel für kleines Federwild, Wachteln u. dergl, sehr geschätzt und häufig abgetragen.]

107. Falco tinnunculus L. $\delta$ ad., $\$$ ad., đิ juv. Oktober, November. Przewalsk.

[Gemeiner Brutvogel im ganzen Gebiet.]

108. Milvus govinda Sykes. ơ ad., 2 \% ad. Juli, August. Przewalsk, Bel-Kara-Szu.

[Gemeiner Brutvogel im ganzen Gebiet.]

109. Pandion haliaëtus L. 2 đ ad. Mai, September. Kok-Dschigde, Koj-Szary.

[Scheint im Gebiete sehr selten zu sein. Ein ô erlegte ich am unteren Ili bei seinem Horste.

110. Haliaetus leucoryphus Pall. o juv., \& juv., 2 juv. ? Mai, Dezember. Ilisk, Przewalsk.

Nacken- und Bauchfedern sind jedoch bei unseren Exemplaren mehr schmutzig gelb.

[Gemein am Ili, wo er allenthalben in Felsen und auf Bäumen, auch im Rohr brütet. Am Issyk-Kul erschienen die Seeadler erst spät im Herbst und auch da nicht eben zahlreich.]

111. Circaetus gallicus (Gm.) oे ad. Mai. Kok-Dschigde. beobachtet.

[Nicht häufig in den Steppen am Ili. Im Gebirge gar nicht

112. Buteo ferox Gmel. đo ad. Mai. Ilisk.

[Sehr zahlreich in allen Steppengegenden.]

113. Buteo menetriesi Bogd. 3 ô ad. Oktober. Karakol-Tal, Koj-Szary, Örtök.

Nach Radde ist jedoch $B$. menetriesi eine Varietät von $B$. tachardus und zwar var. rufus.

[Im ganzen Gebiet, aufser dem Hochgebirge.]

114. Aquila chrysaetus ठ juv. Oktober. Scskili-Tas. 
115. Aquila heliaca Savig. ơ ad., đo ad. ?, $\$$ ad. ? September, Oktober. Santas, Koj-Szary.

[Nicht sehr zahlreich. Die erlegten Vögel scheinen auf der Wanderschaft gewesen zu sein. Als Brutvogel wurde $A$. hel. nicht konstatiert.]

116. Accipiter nisus L. đ’ ad., 2 đ juv. September, Oktober, November. Akszu-Quellen, Przewalsk.

Das $\delta \mathrm{ad}$. hat eine auffallend rostrote Unterseite, die Querbänderung derselben ist stark verschwommen, was wahrscheinlich eine Folge des hohen Alters ist.

117. Astur palumbarius L. đajuv., juv. ? Oktober, Februar. Przewalsk.

[Accipiter nisus und Astur palumbarius auf dem Zuge im ganzen Gebiete gemein, in der Waldregion des Gebirges beide häufige Brutvogel. Ast. palumb. ist ein viel benützter Beizvogel „niederen Fluges" für Hasen, Fasanen u. dergl. Jagd.]

118. Circus cyaneus L. ô ad., ad. \& ?, 2 \& juv. Juli, Jänner. Kacska-Szu, Przewalsk.

Das $\%$ ad., Juli, Kacska-Szu wurde von Almási als $\$$ bestimmt; es hat aber genau das Gefieder eines od ad., er meint es sei dies ein Fall von Hahnfedrigkeit, immerhin ist es möglich, dafs ein Irrtum bei der Geschlechtsbestimmung vorliegt.

119. Circus macrurus Gmel. 2 o juv. August. Belkara-Szu.

120. Circus aeruginosus L. 2 क ad., o juv. Mai, Juni, Oktober. Koj-Szary, Ilisk.

[Die Weihen sind im garzen Gebiet gemein. C. macrurus brütet auf dem Boden in Blössen des Fichtenwaldes bis zu $2500 \mathrm{~m}$ Seehöhe.

121. Gyps himalayensis Hume. o ad., 2 ad. ? August, Dezember. Ecskili-Tas, Przewalsk, Karakol-Tal.

[Sehr häufig im ganzen Hochgebirge.]

122. Turtur monachus L. \$ juv. ? Juli. Karkara.

Nach der dunkeln Färbung der Dunen am Kopf und des übrigen Gefieders zu urteilen, ist das mir vorliegende Exemplar ein junges Tier.

[Häufiger Brutvogel der Waldregion. Sämtliche erlegten und beobachteten Vögel sind auffallend dunkel, besonders an der Halskrause.]

123. Turtur ferrago Eversm. 2 od., 2 ㅇa., 1 ad. ? April, Juni, Juli, August. Karakol-Tal, Ilisk, Karakol-Bas.

[Recht zahlreich an waldigen Stellen der Niederung, aber fast ebenso häufig in den Waldtälern des Tiën-Schan, wo sie bis etwa $3000 \mathrm{~m}$ Meereshöhe als Brutvogel hinaufsteigt.

124. Columba livia unicolor Brehm. 3 ơ ad., 2 \& ad. Juli. Przewalsk.

Von Brehm zuerst im Vogelfang p. 256 für Nubien, von Almásy in Aquila V. p. 160 für die Dobrudscha konstatiert. 2 
typische Exemplare und zwar das von Almásy besprochene ơ und ein đ juv. von Mokro, Mai 1887, beide im Bosn. herzeg. Landesmuseum in Sarajevo, stimmen mit unseren 5 Exemplar sehr gut überein, die ich hier zum erstenmal für den Tienschan konstatiere, wo sie zugleich mit $C$. $l$. typica weit ab von jeder menschlichen Ansiedlung sehr häufig vorkommen und infolge ihrer grofsen Scheuheit nur schwer zu erlegen sind. Almásy versichert bestimmt, dafs $C$. l. unicolor keine verwilderte Haustaube ist; meine Meinung geht dahin, dafs $C$. $l$. unicolor eine melanotische Variation von C. l. typica ist und in manchen Gegenden letztere vertritt. An C. l. typica konnte ich sehr bäufig eine schwarze Fleckung des Mantels und der Scapularien wahrnehmen.

125. Tetrao tetrix viridanus Lor. 3 .. ad., 2 웅., ơ juv. September, Oktober. Türgen, Örtök, Przewalsk.

[Ungemein häufig in der unteren und mittleren Nadelwaldregion.]

126. Tetraogallus himalayanus Gray. ㅇa․, juv. ? Juli, August. Kacska-Szu, Ajuk-Tascz.

[Fehlt an geeigneten Stellen im Gebirge nirgends.]

127. Coturnix coturnix L. 2 o ad., 2 \% ad. Oktober. Koj-Szary. 2 Käfigvögel.

[Überall zahlreich, auf dem Zuge stellenweise in grofsen Mengen. Brütet in der Nähe von Przewalsk auf hochgrasigen Alpenwiesen in der Waldregion bis zu $2600 \mathrm{~m}$ Meereshöhe.]

128. Perdix daurica Pall. 2 क ad., 2 이. Oktober. Przewalsk.

[Im Tekkes-Tal und Issyk-Kul-Becken nicht selten, auf den Hochsteppen des Sary-Dschasgebietes (über $3000 \mathrm{~m}$ ) sehr häufig. In der Iliniederung nicht beobachtet.]

129. Caccabis chukar Gray. 4 o ad., 6 ㅇa., 2 ô juv., 1 juv. ? Juli, September, Oktober. .Boguty-Tau, Karagaity, Kapkak, Akszu-Quellen, Bir-Bas, Örtök, Dsetiguz, Przewalsk.

[Gemein an geeigneten Stellen im ganzen Gebiet, im IliCañon sowohl wie auf den Hochsteppen des Gebirges auf 3000 und $3600 \mathrm{~m}$ Seehöhe.

130. Phasianus mongolicus Brandt. 4 ô ad., ㅇa․ Mai, Oktober, November. Ak-Togoi, Utogoi, Koj-Szary, Przewalsk.

[Der einst im Ssemirgetus sehr häufige Fasan ist heute infolge starker Verfolgung der Zahl nach stark reduziert. An geeigneten Stellen findet er sich aber überall noch vor, von der Balchasch-Niederung angefangen bis in die Laubdjungels des Tekkes-Tales und die Buchsteppen am Issyk-Kul.]

131. Herodias alba L. 2 ô ad., ô juv. Oktober. Koj-Szary.

[Seltener Brutvogel am unteren Ili. Auf dem Herbstzuge nicht selten am Issyk-Kul.]

132. Ardetta minuta L. o ad. Mai. Ak-Szu-Bach. 
133. Botaurus stellaris L. ad. ? Dezember. Przewalsk.

[Die grofse und kleine Rohrdommel kommen nur sporadisch in den überhaupt auffallend vogelarmen Rohrsümpfen des unteren Ili vor. Diese, wie die meisten übrigen Vertreter der Sumpfund Wassersäume, fanden sich weit häufiger während des Herbstzuges am Issyk-Kul ein, als zur Brutzeit in den weiten Rohrund Sumpfländerein der Balchasch-Niederung, die doch dem Anscheine noch die günstigsten Lebensbedingunger. für die Vogelwelt bietet.]

134. Pterocles arenarius Pall. ô ad. Juli. Temirlik-Tal.

[Sehr zahlreich am unteren Ili. Einige kleine Flüge halten sich auf den Steppenhügeln Bir-basch in der Nähe von Przewalsk auf.]

135. Otis tetrax L. 1 juv.

[Nicht eben seltener Brutvogel in den Steppen nahe von Ilisk. Mitte Mai war die Balz in vollem Gange.

136. Fulica atra L. ad. ? Jänner. Przewalsk.

[In den Sümpfen und Rohrichten des Ili überhaupt nicht beobachtet. Auf dem Herbstzuge erschienen grölsere Scharen auf dem Issyk-Kul.]

137. Rallus aquaticus L. † ad. März. Przewalsk.

138. Zapornia parva Scop. 5 ad. April. Tjub.

139. Vanellus vanellus L. ¿ juv., ad. ? August. Przewalsk.

140. Aegialitis dubia Scop. 2 à ad. Mai, Juni. Ilisk, Talgara.

[Rallus, Zapornia, Vanellus und Aegialitis in den prachtvollen Sumpfgegenden des Ili wenig oder gar nicht beobachtet, erschienen sie mehr oder weniger häufig während des Herbstzuges an den Ufern des Issyk-Kul.]

141. Glareola pratincola L. ô ad. Mai. Ilisk.

[Ziemlich häufig in der Umgebung von Ilijsk. Auch mehrere Brutkolonien wurden dort besiedelt.]

142. Ibidorhynchns struthersi Vig. 2 oे ad., $\$$ ad., o juv. $\$$ juv. Juli, Oktober. Karkara, Karakol, Karakol-'Ial.

[Zwischen 2000 und $3000 \mathrm{~m}$ Meereshöhe in einzelnen Paaren auf allen geeigneten Stellen, d. h. überall da, wo die Bergbuchen weite Schattenbänke ausgebreitet haben. In der ersten Hälfte Juli waren die Jungen zum gröfsten Teile noch kaum flügge.]

143. Tringa temmincki Leisl. 3 ơ ad., $\%$ ad., ad. ? Mai, September. Ilisk, Bel-Kara-Szu, Issyk-Kul.

144. Philomachus pugnax L. $\&$ juv. Oktober. Koj-Szary.

145. Tringoides hypoleucus L. ऽ ad., $\$$ ad. August, November.

Karakol-Tal, Przewalsk.

[Brutvogel im Tiën-Schan.] 
146. Totanus ochropus L. के ad., $\%$ ad. August, September. Karagaity, Terskei-Ala-Tan.

[Ziemlich häufig in den Flufsmündungen am Westufer des Issyk-Kul.]

147. Totanus fuscus L. ㅇ ad. Oktober. Koj-Szary.

[Brutvogel am Ili; während des Herbstzuges häufig am Issyk-Kul-Ufer.]

148. Totanus calidris L. đ juv. Juli. Karkara.

[Häufiger Brutvogel. am Ili.]

149. Limosa lapponica L. o ad. September. Issyk-Kul.

[Einige Exemplare während des Herbstzuges; scheint nicht häufig zu sein.]

150. Gallinago solitaria Hodgs. ô ad. Oktober. Bir-Bas (Örtök). 「Auf den Syrts des Tiën-Schan Brutvogel hochgelegener Quellenmoore; nicht eben selten. Im Spätherbste an den Zuflüssen des Issyk-Kul, wo sie in der Nähe des Sees überwintern.]

151. Gallinago gallinago L. 2 o ad. August. Przewalsk.

152. Cygnus olor Gm. ad. ? Dezember. Przewalsk.

[- Brutvogel der Balchasch-Niederung; im Winter ziemlich häufig am Issyk-Kul. - ]

153. Cygnus musicus Bechst. juv. ? Februar. Przewalsk.

[Sehr vereinzelt in den Rohrsümpfen am Ili gesehen wo er zu brüten scheint; im Laufe des September erscheinen die Singschwäne erst vereinzelt, dann immer zahlreicher am Issyk-Kul, wo sie in grofsen Mengen - zu vielen Tausenden - überwintern.]

154. Casarca casarca L. $\&$ juv. Juli. Karakol-Tal.

[Brutvogel des ganzen Gebietes; scheint sich allen Lebensbedingungen anzupassen, da wir sie - vom Kaspi an - überall beobachteten, in den Steppen und Saksaul-Wüsten, am mittleren und unteren Ili sowohl, wie an den Waldbächen und auf den Hochsteppen des Tiën-Schan, also vom Spiegel des Kaspi an bis auf über $3000 \mathrm{~m}$ Meereshöhe.]

155. Anas crecca L. ơ ad., ad. ? August, September. Terskei-Ala-Tan.

[Am Ili und am Issyk-Kul nicht selten.]

156. Netta rufina Pall. 5 б ad., 2 워 ad. Mai, Dezember, Jänner. Kok-Dschigde, Przewalsk.

[So ziemlich die häufigste Ente am unteren Ili während der Brutzeit. Im Winter zu vielen Tausenden am Issyk-Kul.]

157. Querquedula circia L. \% ad. September. Naryn.

[Brutvogel am mittleren Ili.]

158. Fuligula nyroca Güldenst. đo ad., ㅇa ad. Mai. Ilisk.

[Ziemlich gemein am Ili.]

159. Fuligula clangula L. $\%$ ad. Jänner. Przewalsk. 
[Am Ili zu so später Jahreszeit gesehen, dafs ihr Brüten dortselbst nicht unwahrscheinlich erscheint. Im Winter ziemlich häufig am Issyk-Kul.]

160. Fuligula ferina L. ఫ ad., \& ad. Jänner. Przewalsk.

[Brutvogel am Ili, im Winter zahlreich am Issyk-Kul.]

161. Erismatura leucocephala L. $\%$ ad. Oktober. Koj-Szary.

[Recht häufiger Brutvogel am Ili. Im Herbst zahlreich am Issyk-Kul.]

162. Mergus albellus L. $\$$ ad., 1 pull. ? August, Jänner. TerskeiAla-Tau, Przewalsk.

[Häufiger Wintergast am Issyk-Kul.]

163. Pelecanus onocrotalus Gmel. \& ad. Mai. Kok-Dschigde. [Sehr gemein am Ili.]

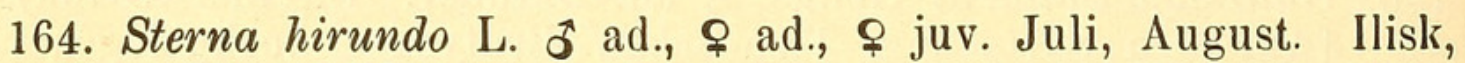
Przewalsk.

165. Larus minutus Pallas. $\$$ ad. August. Bel-Kara-Szu.

166. Larus ridibundus L. 2 o ad., $\$$ ad., ad. ? Mai, September. Ilisk, Koj-Szary.

Ein $\widehat{\sigma}$ ad. und ad. ? sind dadurch bemerkenswert, dafs das Schwarz an den ersten 3 Schwungfedern von innen und aufsen zusammenfliefst, so dafs bei ad. ? an den ersten 2 Schwungfedern blos ein $2 \mathrm{~cm}$ langes weifses Streifchen $\mathrm{zu}$ sehen ist, bei dem ठ ad. ist dieser Streifen länger, was Saunders im Cat. of Birds Brit. Museum XXV. p. 213 an 2 Exemplaren aus Dinapur erwähnt.

167. Larus argentatus cachinans Pall. $\%$ ad. September. Koj-Szary.

['Trotz des enormen Fischreichtums aller Gewässer des Gebietes, sind die Möven und Seeschwalben relativ spärlich vertreten. Am unteren Ili bis in's Delta hinein trafen wir nur ganz vereinzelte Möven an; verhältnismiäfsig zahllreich fanden wir diese Arten L. ridibundus und St. hirundo in den Reisfeldern der Dunyanen am mittleren Ili bei Tschilik und Tscharym. Das Exemplar von Larus minutus traf ich am 29. August 1900 bei einigen kleinen Steppenseen auf der Hochfläche des Sary-Dschas (circa $3500 \mathrm{~m}$ ), inmitten von Kettenzügen von durchschnittlich $4000 \mathrm{~m}$ mittlere Höhe. Dieses vereinzelte Exemplar dürfte die Tiën-Schan-Ketten auf dem Zuge von Ost nach Westen überflogen haben.]

168. Podiceps nigricollis Brehm. 2 đ̄ ad. Oktober, Jänner. KojSzary, Przewalsk.

[Am Ili nicht seltener Brutvogel.]

169. Podiceps cristatus L. is ad. im Winterkleid. September. Koj-Szary.

[Häufiger Brutvogel der Balchasch-Niederung. Im Winter beide Taucher nicht selten am Issyk-Kul. - ] 


\section{$2 \mathrm{BHL}$ Biodiversity Heritage Library}

1906. "Ein Beitrag zur Ornis des Tianschan." Journal fu

r Ornithologie 54, 411-428. https://doi.org/10.1007/bf02089281.

View This Item Online: https://www.biodiversitylibrary.org/item/104732

DOI: https://doi.org/10.1007/bf02089281

Permalink: https://www.biodiversitylibrary.org/partpdf/142619

\section{Holding Institution}

Smithsonian Libraries

\section{Sponsored by}

Biodiversity Heritage Library

\section{Copyright \& Reuse}

Copyright Status: Public domain. The BHL considers that this work is no longer under copyright protection.

This document was created from content at the Biodiversity Heritage Library, the world's largest open access digital library for biodiversity literature and archives. Visit BHL at https://www.biodiversitylibrary.org. 